\title{
DESARROLLO PSICOMOTRIZ EN LA INFANCIA Y DESEMPEÑO DOCENTE
}

\author{
PSYCHOMOTRIZ DEVELOPMENT IN CHILDHOOD \\ AND TEACHING PERFORMANCE
}

\author{
Edgar Froilán Damián Núñez ${ }^{1}$ \\ Zoila Noemí Merino Acosta ${ }^{2}$ \\ Neptali Antony Reyes Cabrera ${ }^{1}$ \\ Nalda Guadalupe Damián Nuñez ${ }^{1}$
}

1 Universidad Nacional Mayor de San Marcos, Perú. edamiann@unmsm.edu.pe

2 Universidad Estatal Península de Santa Elena, Ecuador.

\section{RESUMEN}

La presente investigación tiene como propósito establecer si existe relación entre el desarrollo psicomotor de los estudiantes de educación inicial (preescolar) y el desempeño docente, a partir de los roles que deben cumplirse para que las competencias de los niños se desarrollen. Con este propósito, se elaboraron fichas de observación, para ambas variables, que fueron aplicadas a 10 docentes y 90 estudiantes de Instituciones Educativas Iniciales del distrito de Independencia (pertenecientes a la UGEL $N^{\circ} 02$ en 2014). Los resultados del análisis estadístico demostraron que no existe relación entre ambas variables, con un coeficiente de correlación de Pearson de 0,319, a pesar que el coeficiente de significancia bilateral fue de 0.02 .

Palabras clave: Psicomotricidad, infancia, desempeño, docente.

\begin{abstract}
The purpose of this research is to establish whether there is a relationship between the psychomotor development of students in early childhood education (pre-school), and the teaching performance of initial education roles for children's competencies. For this end, observation cards were elaborated for both variables, which were applied to 10 teachers and 90 students from the initial educational centers of the district of Independencia (belonging to UGEL $N^{\circ} 02$ in 2014). The results after the statistical analysis demonstrated that there is no relationship between both variables with a Pearson correlation coefficient of 0.319 despite the fact that the coefficient of bilateral significance is 0.02.r.
\end{abstract}

Key words: Psychomotricity, childhood, performance, teacher.

Recibido: 08 de febrero de 2018

Aprobado: 07 de marzo de 2018

Publicado: 20 de junio de 2018

\section{INTRODUCCIÓN}

La actividad física es una manifestación común en el hombre. Este la tiene desde su aparición en la tierra, con distintos objetivos, debido a que la realizaba para conseguir alimento, para luchar, para movilizarse, entre otras actividades. Así, siempre ha estado ligada al hombre y su cultura en toda la historia. Por esa razón, no es raro que en la historia de las civilizaciones se detecten claros vínculos entre la ocupación humana y el ejercicio físico para asegurar la continuidad de la especie humana.

No es hasta el siglo XIX, sin embargo, que se otorgó importancia a las ciencias aplicadas del deporte, la cual se manifestó en publicaciones que recopilaron los juegos en las culturas de Europa y América. Estas demostraron que la antropología clásica no era adecuada para establecer sus formalizaciones científicas, pues la relación del hombre con lo sagrado exige una naturaleza reflexiva constante en todas las 
épocas de la humanidad. Por esa razón, se usó primordialmente para reivindicar la religiosidad(1) en la construcción de la identidad y los valores deportivos en la educación.

La arqueología, en cambio, descubrió numerosas evidencias (representaciones de cacería, lucha y danza) en que puede identificarse el carácter mágico de la actividad física. Por otra parte, gracias a estudios sobre distintas formas de ejercicio físico, se pudieron distinguir elementos entre los pueblos primitivos que indican no solo su aspecto religioso, sino también lúdico, cuasi deportivo(2). Así, muchas culturas expresan su religiosidad mediante la actividad física.

En Perú, se creó la Escuela Nacional de Educación Física mediante el Decreto Supremo 328 , del 6 de setiembre de 1932, en la administración de gobierno del general Luis Sánchez Cerro. Por esa razón, cada año en la referida fecha se conmemora la creación de la primera escuela nacional de educación física, así como, se realiza un homenaje a los docentes de la especialidad. Esto surge después de la Conferencia General de la Organización de las Naciones Unidas para la Educación, la Ciencia y la Cultura, reunida en París el 21 de noviembre de 1978, en que se proclamó la "Carta Internacional de la Educación Física y el Deporte"(3) donde se establece la práctica de estas disciplinas como derecho fundamental.

Es así porque el desarrollo del ser humano, desde su condición de óvulo fecundado hasta que se convierte en adulto, se relaciona con continuas transformaciones físicas. En ese proceso de cambios, el desarrollo físico y el desarrollo psicomotor requieren atención especial en los primeros años de vida, pues en esa etapa se presentan las rápidas y sucesivas transformaciones que tendrán repercusión en su desarrollo integral. Se requiere, por tanto, del desempeño profesional de docentes que mediante el manejo de habilidades, destrezas y conocimientos en la programación de clasesorganicen y ejecuten su trabajo de acuerdo a metas y competencias establecidas.

\section{DESARROLLO TEÓRICO}

El término psicomotricidad tiene dos acepciones básicas:(4) por un lado, hace referencia a la interrelación entre las funciones neuromotrices y psíquicas del ser humano y, por otro, al conjunto de técnicas encaminadas a su desarrollo general que, partiendo de la educación del movimiento, posibilita la correcta interacción entre el medio ambiente y la función simbólica. En la actualidad, la psicomotricidad contempla ambos aspectos.

Así, el desarrollo psicomotor es la base del aprendizaje escolar. La evolución psicomotriz depende fundamentalmente de la maduración neurológica que transita de una fase automatizada (en los primeros meses) a otra receptiva (en el segundo trimestre) y coincide con la mayor capacidad discriminativa de los órganos de los sentidos. Luego sigue una fase de experimentación o adquisición de conocimientos que se prolonga por el transcurso de la vida ${ }^{(5)}$. Este desarrollo se valora a partir de muestras significativas de modificaciones motrices en la conducta adaptativa y personal-social.

Este enfoque, según el Ministerio de Educación ${ }^{(6)}$, utiliza contenidos acordes y relacionados con las ciencias aplicadas que desarrollan no solo las capacidades físicas, sino también: la identidad, la autoestima, el pensamiento crítico y creativo, la toma de decisiones, y la resolución de problemas en contextos de la actividad física y la vida cotidiana. Es decir, que dicho enfoque busca contribuir con el proceso de formación y desarrollo humano integral de los niños para su bienestar y el de su entorno.

La comprensión progresiva y toma de conciencia de sí mismo que el niño desarrolla, en interacción con su entorno y las demás personas, son las que permiten construir su identidad y autoestima. Esto tiene como consecuencia que el infante se desenvuelva por iniciativa propia y disfrute de interiorizar y organizar sus movimientos eficazmente, según sus posibilidades.

El currículo peruano se estructura en base a competencias que se acompañan de referentes para su evaluación formativa. Esto hace sostener que los niños del nivel de educación inicial (orientado a que se conozcan y tomen conciencia de sí mismos) se puedan expresar y desenvolver de manera autónoma mediante su motricidad ${ }^{(6)}$. Es decir, que estos niños podrán lograr, de manera progresiva, la construcción de su imagen y su esquema corporal, a partir de la exploración de sus movimientos, posturas y desplazamientos, durante el juego y la actividad autónoma, en interacción con su entorno.

Aunque estas competencias se desenvuelven de manera autónoma, requieren de condiciones que favorezcan su desarrollo al máximo nivel. En el presente trabajo, se sostiene que los materiales y espacios que requiere el niño para desenvolverse, en la primera etapa, pueden estar a cargo de los padres en casa. Sin embargo, cuando empieza la siguiente etapa 
requiere de los espacios y materiales brindados en el aula con la guía, además, de una imagen docente que cumpla el rol de conciliador. Es decir, que no interfiera con el desarrollo de los estudiantes, pero que pueda y deba guiarlos. Los roles que cumplirá el docente son:(6)

- Posibilitar que los niños jueguen y exploren libremente su cuerpo, así como, hagan uso de su creatividad y posibilidades motrices en actividades relacionadas con distintos movimientos, posturas y desplazamientos, de acuerdo a sus intereses y posibilidades

- Respetar el desarrollo motor autónomo de cada niño: sus posturas, su forma singular de moverse y sus propios ritmos y tiempos. Asimismo, contribuir con la constitución favorable de su identidad y el desarrollo saludable de sus emociones (por lo que no podrá restringirlos, presionarlos 0 apurarlos). Finalmente, promover que los niños puedan medir los riesgos por sí mismos, al momento de realizar sus distintos movimientos, y brindarles la confianza y seguridad necesarias mediante palabras y lenguaje corporal.

- Observar a los niños durante sus juegos y movimientos y prestar más atención al proceso que a la acción en sí misma. Es decir, valorar su esfuerzo, placer y aprendizaje al enfrentar diversos retos motrices.

- Prestar atención a las diferentes sensaciones y/o emociones que los niños expresan mediante gestos, tonos, posturas, movimientos, desplazamientos y juegos para acompañarlos asertivamente $y$ responder a sus necesidades.

Como puede desprenderse de estos roles, el docente es básicamente un observador que respeta y posibilita situaciones favorables para el adecuado desenvolvimiento psicomotriz del niño.

Según el currículo nacional de educación básica (7), un niño se desenvuelve de manera autónoma, mediante su motricidad, cuando evalúa críticamente sus procesos físicos en relación a aspectos sociales, emocionales, afectivos y cognitivos. Asimismo, cuando los vincula con las características de su desarrollo e identidad corporal y demuestra habilidades en la ejecución de movimientos complejos. Así, ese niño está en condiciones de crear, evaluar y apropiarse de prácticas corporales en diferentes contextos y fusionar, además, características expresivas de distintas culturas, incluida la propia. Cabe incidir que esto es consecuencia directa de un conjunto de factores entre los que se encuentra el relacionado a los materiales y espacios adecuados de desenvolvimiento.

Para evaluar el desempeño docente en Perú, se ha implementado (desde los años 20132017) la teoría del monitoreo y desempeño docente que prioriza la evaluación y el desarrollo de competencias. Estas se reúnen en cuatro dominios:(1)

1. Preparación para el aprendizaje de los estudiantes.

2. Enseñanza para el aprendizaje de los estudiantes.

3. Participación en la gestión de la escuela articulada con la comunidad.

4. Desarrollo de la profesionalidad y la identidad docente

Para esta investigación, se han priorizado el primer y segundo dominios por estar directamente relacionados con la planificación y conducción de las sesiones de aprendizaje. Asimismo, se ha establecido específicamente la relación con las siguientes competencias:

Tabla 1. Competencia según el desempeño docente.

\begin{tabular}{|c|c|}
\hline $\begin{array}{l}\text { DESEMPEÑO } \\
\text { DOCENTE }\end{array}$ & COMPETENCIA \\
\hline 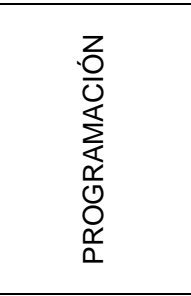 & $\begin{array}{l}\text { Competencia } 2 \\
\text { Planifica la enseñanza de manera } \\
\text { colegiada y garantiza la coherencia } \\
\text { entre los aprendizajes que se } \\
\text { quiere lograr en los estudiantes: el } \\
\text { proceso pedagógico, el uso de } \\
\text { recursos disponibles y la } \\
\text { evaluación, en un programa } \\
\text { curricular en revisión permanente. }\end{array}$ \\
\hline 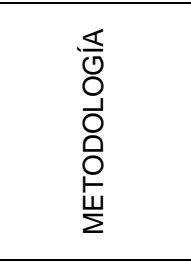 & $\begin{array}{l}\text { Competencia } 3 \\
\text { Crea un clima propicio para el } \\
\text { aprendizaje, la convivencia } \\
\text { democrática y la vivencia de la } \\
\text { diversidad en todas sus } \\
\text { expresiones, con miras a formar } \\
\text { ciudadanos críticos e } \\
\text { interculturales. }\end{array}$ \\
\hline 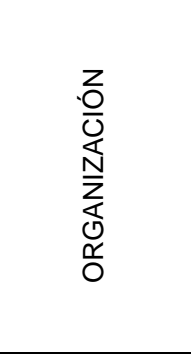 & $\begin{array}{l}\text { Competencia } 4 \\
\text { Conduce el proceso de enseñanza } \\
\text { con dominio de contenidos } \\
\text { disciplinares y uso de estrategias y } \\
\text { recursos pertinentes. Esto para que } \\
\text { los estudiantes aprendan de } \\
\text { manera reflexiva y crítica lo } \\
\text { concerniente a la solución de } \\
\text { problemas relacionados con sus } \\
\text { experiencias, intereses y contextos } \\
\text { culturales. }\end{array}$ \\
\hline
\end{tabular}

El desarrollo psicomotor en las sesiones de aprendizaje se relaciona con el desempeño docente que, según la metodología: planifica, concreta y determina una organización. La metodología que se adecúa y relaciona con el desempeño docente y el desarrollo psicomotriz es la metodología activa. Esta se entiende como una enseñanza centrada en los estudiantes, pues brinda contenidos y actividades de interés relacionados con los 
contextos y saberes de los referidos para que no solo puedan entender y aprender, sino también generar nuevos conocimientos. (2) La metodología activa, utilizada en las sesiones de aprendizaje, cumple con las expectativas del desarrollo psicomotor requerido para el cumplimiento del buen desempeño docente.

\section{MATERIALES Y MÉTODOS}

La investigación sigue un diseño correlacional, pues utiliza el modelo de regresión y correlación lineal de Pearson. Este tipo de estudio está interesado en la determinación del grado de relación existente entre dos variables de interés.

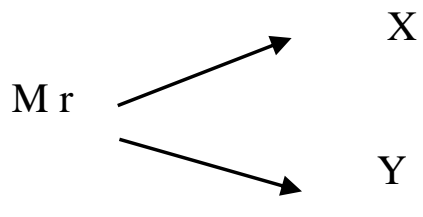

- "M" representa a los participantes del nivel preescolar y sus profesores.
- "X" representa al desarrollo psicomotriz.

- "Y" representa al desempeño docente del nivel preescolar.

- " $r$ " representa al grado de relación entre las variables.

Se tomó el coeficiente de correlación lineal simple de Pearson representando por la siguiente igualdad:

$$
r=\frac{N\left(\sum X Y\right)-\left(\sum X\right)\left(\sum Y\right)}{\sqrt{\left[N\left(\sum X^{2}\right)-\left(\sum X\right)^{2}\right]\left[N\left(\sum Y^{2}\right)-\left(\sum Y\right)^{2}\right]}}
$$

La población está compuesta por todos los profesores y participantes de las instituciones educativas de nivel preescolar del distrito de Independencia.

La muestra fue tomada de manera conveniente a nueve estudiantes y un profesor de cada uno de las diez instituciones educativas elegidas.

Para la recolección de datos se utilizó la ficha de observación que contiene reactivos en base a los indicadores de la variable independiente y dependiente, según sea el caso, como se muestra en la Tabla 2:

Tabla 2. Operacionalización de variables

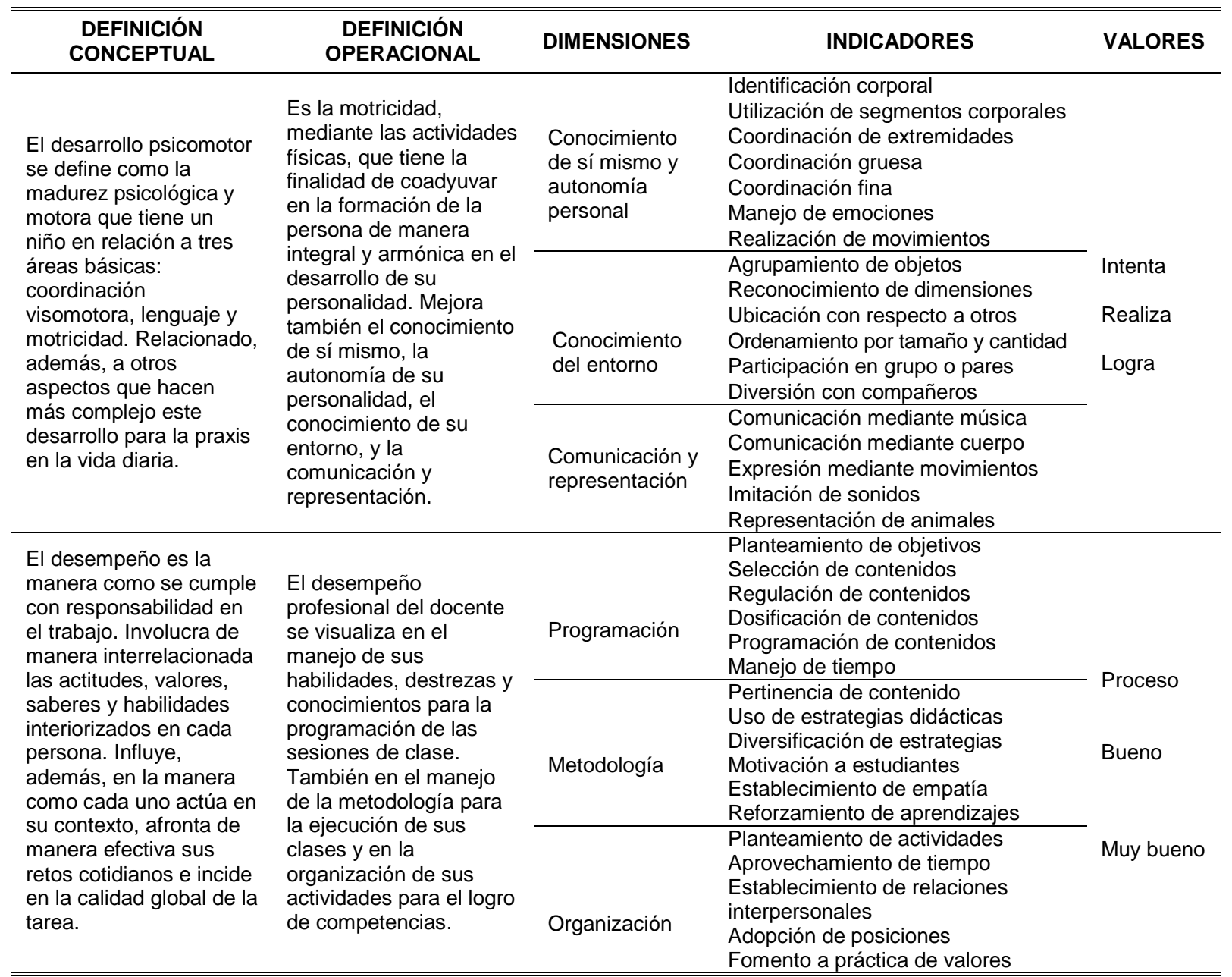


Para el análisis de los datos, se utilizó el software estadístico SPSS en su versión 22.

\section{RESULTADOS}

La investigación se realizó en Instituciones Educativas Iniciales del Distrito de Independencia (UGEL Nº2, 2014). Se evaluó a docentes y estudiantes de nivel preescolar, con el objetivo de determinar la relación entre las variables "Desempeño docente" y "Desarrollo psicomotriz". La figura 1 muestra los resultados obtenidos.

Sobre el desempeño motriz en el nivel preescolar, se observa que el 48,89 \% de estudiantes evaluados, o sea la mayoría, "realiza" actividades psicomotrices sin mayor dificultad, mientras el $14.44 \%$ solo "intenta" realizar actividades psicomotrices (figura 2).

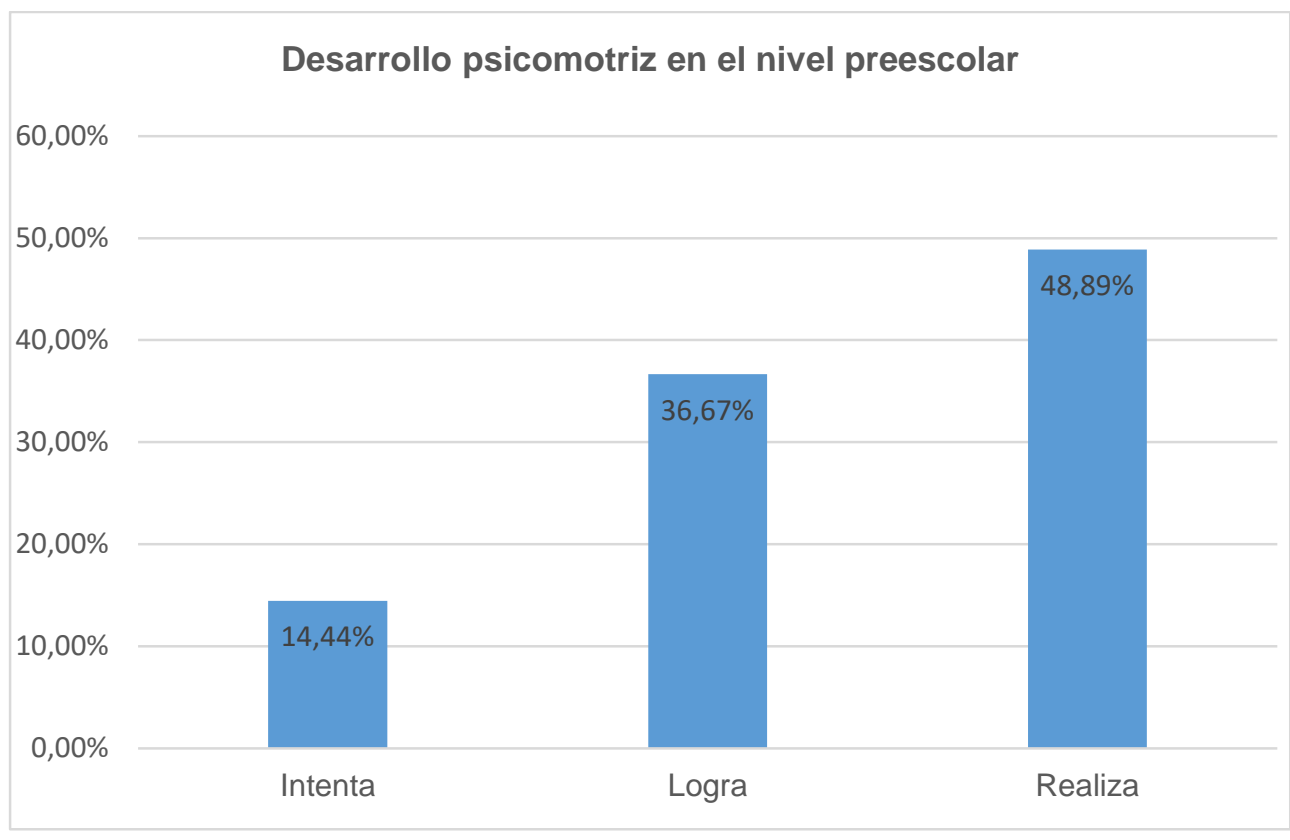

Figura 1. Desarrollo psicomotriz en el nivel preescolar.

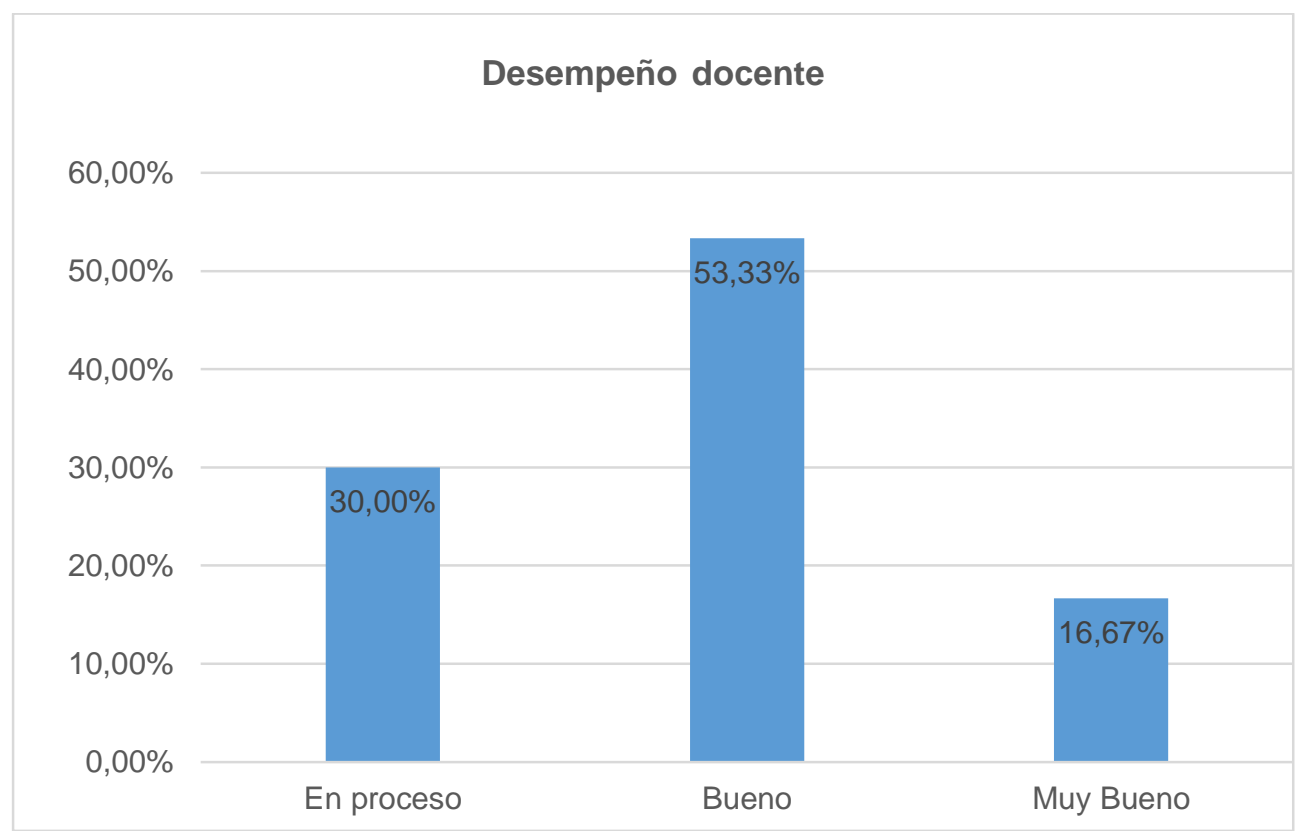

Figura 2. Nivel del desempeño docente.

Sobre el desempeño docente, se observa que el $53.33 \%$ de docentes ha alcanzado el nivel de "bueno", mientras solo un $16.67 \%$ el de "muy bueno". También que el $30,00 \%$ de 
docentes se encuentra en proceso de desarrollo y, por tanto, el $83.33 \%$ no ha llegado al nivel de "muy bueno".

Al realizar el análisis de correlación, se observa como principal resultado el expresado en la tabla 2.

Tabla 2. Correlación entre variables.

\begin{tabular}{cc}
\hline \hline Coeficiente de correlación & 0,319 \\
\hline Sig. (bilateral) & 0,002 \\
\hline
\end{tabular}

Así, a pesar que el coeficiente de significancia bilateral es menor que 0.005 (0.005>0.002), el coeficiente de correlación se encuentra a una distancia más que considerable del 1 , por lo que se concluye que no existe correlación entre ambas variables.

\section{CONCLUSIONES}

- No existe asociación entre el desempeño docente y el desarrollo psicomotor de los estudiantes en el nivel inicial (preescolar), pues los profesores cumplen roles de observadores, posibilitadores y de respeto hacia los referidos. Esto resuelve que no determinen, sino que solo promuevan el desarrollo de sus habilidades.

- El desempeño docente muestra un desarrollo heterogéneo y desigual. Así, registra un bajo porcentaje de desarrollo en la categoría de "muy bueno", mientras en la categoría de "bueno" se concentra la mayor parte de la muestra

- Por su excepcional dinamismo, los estudiantes de preescolar muestran, con algunas excepciones, un elevado desempeño y tienden a manifestar su desarrollo psicomotor de manera autónoma.

\section{REFERENCIAS}

1. Ministerio de Educación del Perú. Marco del buen desempeño docente. Segunda edición. Lima: MINEDU; 2014.

2. Macfarlan, A. Book of american indian games. New York: Bombay; 1958.

3. UNESCO. Carta Internacional de la Educación Física y el Deporte. París: UNESCO; 1978.

4. Maganto, C. Desarrollo físico y psicomotor en la etapa infantil. San Sebastián: Facultad de Psicología-UPV; 2011.

5. Jiménez, M. Intervención educativa en el ámbito psicomotriz. Coruña; 2004; p. 1-15.

6. Cachán, C. R. Educación, religión y deporte: fundamentos, valores y retos del futuro. Río de Janeiro: 2015; p. 5-18.

7. Ministerio de Educación del Perú. Currículo Nacional de Educación Básica. Lima: MINEDU; 2016.

8. Ministerio de Educación del Perú. Programa Curricular de Educación Inicial Lima: MINEDU; 2016.

9. Galvez, R. E. Metodología activa. Lima: Santillana; 2013. 